\title{
The Interface Between the Practice of Medical Genetics and Human Genetic Research: What Every Genetic Counselor Needs to Know
}

\author{
Dorene S. Markel ${ }^{1,3}$ and Beverly M. Yashar ${ }^{2}$
}

Genetic counselors have historically used human genetic research as an advanced information resource for their patients. Most commonly, this has been via access to information provided by gene identification studies in advance of commercial testing. More recently genetic counselors have been participating in human genetic research studies as part of the investigative team. This review provides a framework to help genetic counselors in research and clinical practice understand the historical perspectives, ethical principles, and federal regulations that govern the current practice of human subject research. Special consideration is given to the IRB process and unique issues in human genetic research. This overview is intended to help improve the ability of genetic counselors to act as advocates for their patients.

KEY WORDS: Institutional Review Board (IRB); human subject research; genetic research; Belmont Report; genetic counseling; genetic counselor; ethics, research regulations.

\footnotetext{
${ }^{1}$ Center for the Advancement of Clinical Research, Department of Medical School Administration, University of Michigan, Ann Arbor, Michigan.

${ }^{2}$ Departments of Human Genetics and Ophthalmology and Visual Sciences, University of Michigan, Ann Arbor, Michigan.

${ }^{3}$ Correspondence should be directed to Dorene S. Markel, MS, MHSA, The University of Michigan Medical School, Center for the Advancement of Clinical Research (CACR), Domino's Farms, 24 Frank Lloyd Wright Drive, Lobby M, Ann Arbor, Michigan 48109-0738; e-mail: markel@umich. edu.
} 


\section{LIST OF ABBREVIATIONS}

\begin{tabular}{ll}
\hline ASHG & American Society of Human Genetics \\
DHHS & The Department of Health and Human Services \\
FDA & The Food and Drug Administration \\
IRB & Institutional Review Board \\
NCPHS & $\begin{array}{l}\text { National Commission for the Protection of Human } \\
\text { Subjects of Biomedical and Behavioral Research }\end{array}$ \\
NIH & National Institutes of Health \\
NSGC & National Society of Genetic Counselors \\
OHRP & Office of Human Research Protections \\
45 CFR 46 & Code of Federal Regulations Title 45-Public Welfare, \\
& Part 46-Protection of Human Subjects \\
\hline
\end{tabular}

\section{INTRODUCTION: THE INTERFACE BETWEEN GENETIC COUNSELING PRACTICE AND GENETIC RESEARCH}

The completion of the human genome project has increased our ability to genetically define disease and widely expanded research studies utilizing genetic information. Although enhanced technological ability and increased genetic research will lead to the increased availability of clinical genetic tests, attainment of this endpoint will require intermediate stages where patients and families with genetic traits or conditions will be requested to participate in genetic research studies. A patient or family's involvement in these studies may be purely altruistic with the hope that the knowledge gained will someday assist others. However, their participation in research may be with the intent of personal direct benefit by gaining access to information that would not be otherwise available. This information may be of future interest only, such as an individual's projected risk to develop a particular disease or condition, or the information may have critical immediate value to the patient or family, such as the possible prenatal diagnosis of a fetus with a congenital anomaly or disease. Genetic counselors are often involved in both of these scenarios as part of their routine practice.

Being the interface between the clinical and research worlds is not new to the field of genetic counseling. Since the early days of the genetic counseling profession, genetic counselors have sought out research labs to help provide information not yet commercially available to their patients and assisted researchers in identifying patients and families that are essential for the success of genetic research studies. Although the field of genetic counseling could have limited its practice to only those genetic tests that are commercially available and thus appropriately validated, this has not been genetic counseling practice and would be judged by most genetic counselors as not providing the best service possible to their patients. 
This, however, is not a role typically filled by other allied health professions or physicians and brings with it responsibilities for which there are limited clinical practice referents.

Playing the intermediary role between their patients and research laboratories is viewed as an essential part of the professional responsibilities of a genetic counselor; however, genetic counselors must be aware of the added ethical and regulatory responsibilities that are involved in human subject research. Although there are many genetic counselors that are part of teams working on genetic research projects and are well experienced in these areas, they are in the minority. In the 2002 National Society of Genetic Counselors (NSGC) Professional Status Survey, $30 \%$ of genetic counselors indicated that their primary role was "research/study coordination" (NSGC, 2002). This percentage remains relatively unchanged from the $31 \%$ reporting "research" as their primary role in the 2000 NSGC Professional Status Survey. This places "research" third in the primary roles reported by genetic counselors behind "clinical" $(86 \%)$ and "teaching/education/supervising students" $(56 \%)$. Thus the majority of genetic counselors have not had the direct opportunity to learn about the ethical and regulatory issues involved in human subject research.

Concomitant with the growth in human genetic research, the regulations that govern the utilization of human subjects or their tissue/DNA in research are being reexamined, more stringently interpreted, and applied. Institutions around the country and the world have increased their scrutiny of human subject research and expanded the educational requirements for those involved in this research to protect the privacy, safety, and trust of those that choose to participate in research studies (National Institutes of Health [NIH], 2001). Many of these institutional improvements have come in response to federal sanctions against institutions that have not fully protected the rights of human subjects (Office of Human Research Protections [OHRP], 2003b). The Department of Health and Human Services (DHHS) Office of Human Research Protections website provides on-line access to "determination letters" sent to institutions around the country sighting both major and minor deficiencies in their human subject protection systems. These deficiencies have in some cases resulted in the partial or complete shutdowns of all federally funded human subject research activity at these institutions until improvements and resolutions were approved by OHRP (OHRP, 2003b). Such sanctions have been costly to institutions not only in the loss of research revenue, but also because of the negative impact on the institution's reputation.

The complex issues in the regulation and practice of human subject research have a broad impact not only on research genetic counselors who work as members of research teams, but also on those who facilitate the involvement of their patients in research studies. It is essential for those with both direct and indirect research roles to be aware of the ethical principles and regulations in human subject research. The purpose of this article is to provide a framework for considering how and when 
patients should be involved in research and to facilitate the genetic counselor's understanding of their responsibilities when introducing these options.

\section{What Is Human Research and What Ethical Principles Govern Its Conduct?}

The ethics of human subject research and the federal regulations that oversee this research have evolved over the past 40 years. In 1974, Congress passed the National Research Act. This Act created the National Commission for the Protection of Human Subjects (NCPHS) of Biomedical and Behavioral Research, which wrote the Ethical Principles and Guidelines for the Protection of Human Subjects of Research (commonly known as the Belmont Report). This report was published in the Federal Register in 1979. The Belmont Report describes the ethical principles that form the foundation of the federal regulations for the protection of research subjects. These ethical principles were translated into formal federal regulations by the Department of Health and Human Services (DHHS) and the Food and Drug Administration (FDA) and adopted in 1981 as the Code of Federal Regulations Title 45-Public Welfare, Part 46-Protection of Human Subjects (more commonly known as 45 CFR 46). These regulations set the standard for the conduct of all federally funded research involving human subjects, but these standards are typically applied by most institutions to all human subject research regardless of the funding source.

To know when the regulations and ethical concerns that pertain to human subject research apply, one must first understand how human research is defined and what separates it from medical practice. Differentiating research from practice is often difficult because they can occur simultaneously. The Belmont Report, published in 1979, provides the ethical principles and guidelines for the protection of human subjects in research. In doing so it also distinguished between clinical research (including biomedical and behavioral specialties) and accepted clinical practice (Table I; NCPHS, 1979). When a clinician departs in a significant way from

Table I. Distinguishing Clinical Practice and Clinical Research

\begin{tabular}{llc}
\hline & \multicolumn{1}{c}{ Clinical practice } & Clinical research \\
\hline Purpose & $\begin{array}{c}\text { Promote patient well-being via } \\
\text { diagnosis, treatment, or therapy } \\
\text { Direct benefit; geared towards the } \\
\text { individual; high }\end{array}$ & $\begin{array}{c}\text { Contribute to generalizable } \\
\text { knowledge } \\
\text { Possible but generally unknown; } \\
\text { focused on knowledge acquisition }\end{array}$ \\
likelihood/expectation of success & Value of practice was previously \\
Methodology & Experimental protocol; hypothesis \\
Goals & $\begin{array}{c}\text { Short term and long term are } \\
\text { equivalent and both patient driven }\end{array}$ & $\begin{array}{c}\text { Short term: knowledge driven; long } \\
\text { term: patient driven }\end{array}$ \\
\hline
\end{tabular}


standard or accepted practice, this deviation does not, in and of itself, constitute research. The fact that such a deviation is experimental, in the sense that it is new or untested, does not automatically categorize it as research. In the best practice of responsible medicine, major deviations should be "tested" through a formal research process before they are accepted as a standard of clinical care; however not all therapies are investigated in a rigorous clinical research trial before implementation (NCPHS, 1979). Although research is designed to gain generalizable knowledge, it is possible in some types of studies for direct benefit to subjects to also be obtained.

What constitutes a human subject? In our society there is much controversy about what is "human." The federal regulations defined a human subject for the purpose of determining when the federal regulations governing human subject research would apply $(45 \mathrm{CFR} 46,2001)$. This definition of human subject includes any living individual or fetus about whom an investigator (whether professional or student) conducting research obtains (1) data through intervention or interaction with the individual or (2) identifiable private information. Thus by definition, deceased individuals are not human subjects and research on the deceased, their tissue, or their information is not covered under the federal regulations for human subject research. In addition, public information studied or observed in the conduct of research involving living persons is also not covered by these federal regulations. An example of this type of research would be an observational study of anonymous individuals engaged in public behavior. This type of study is not often found in medical settings and thus will not be further discussed in this paper.

The Belmont Report describes three basic principles central to the ethics of human subject research. They are (1) respect for persons, (2) beneficence, and (3) justice. These principles are the foundation of the federal regulations that protect human subjects (NCPHS, 1979). There are also national and state laws and regulations, as well as international and professional codes that guide and evaluate the conduct of investigators (ICH, 1996; 21CFR312, 2002; World Medical Association, 2002 ). The Code of Ethics of the National Society of Genetic Counselors (NSGC) reflects these principles as well (NSGC, 1992).

Respect for persons incorporates two ethical convictions: first, that individuals should be treated autonomously, and second, that persons with diminished autonomy are entitled to increased protections. The process of providing information and obtaining informed consent in the conduct of research is a critical aspect of showing respect for the subject. This also requires that the subject have the ability to make a free and willing choice to participate in the research. The concept that some individuals have diminished autonomy is central to the definition of a "vulnerable population" being those groups that may contain individuals who have limited autonomy and thus would not be able to fully participate in the informed consent process. Such groups include children, mentally or cognitively impaired individuals, and prisoners. Special considerations apply when research involves persons from any of these populations. In addition, pregnant women are 
also recognized by the federal regulations as a vulnerable population due to the additional health concerns during pregnancy and because of the need to avoid unnecessary risk to the fetus.

Beneficence, as defined in the Belmont Report, is an obligation to both (1) do no harm and (2) maximize possible benefits and minimize possible harms. The principles of beneficence are translated into the federal regulations as a requirement to perform risk-benefit assessments (NCPHS, 1979). The term risk refers to the possibility that harm may occur and the severity of the potential harm. The term benefit is used in the context of research to mean something of positive value to health or welfare. Accordingly, risk-benefit assessments are concerned with the probabilities and magnitudes of possible harms compared with anticipated benefits. Risks and benefits of research may affect the individual subjects, the families of the subjects, special groups within society, and even the society at large. Beneficence requires that we protect against the risk of harm to study volunteers and that we are also concerned about the potential loss of benefits that could be accrued as part of standard clinical care. Often determining the balance between potential personal risk to the subject and potential societal benefit constitutes a key ethical dilemma in research ethics.

Justice is the principle that requires fairness in distribution. An injustice occurs when benefits to which a person is entitled are denied without good reason or when burdens are imposed unduly. For example, the selection of research subjects should not be drawn inequitably from certain classes or groups in society simply because of their easy availability or compromised position. Likewise the justice principle requires that research should not unduly involve persons or groups of persons that are not likely to be among the beneficiaries of the potential positive applications of the research. The principle also requires inclusion of diverse populations/groups so that they may benefit from the findings of the research. In the regulations, the principle of justice requires review of procedures for the selection of subjects and the outcome of those procedures.

\section{Who Protects Human Subjects and What Is Their Locus of Authority?}

Current federal regulations for the protection of human subjects in research were directly derived from the ethical principles of the Belmont Report discussed above. In 1991, 17 federal departments and agencies adopted a common set of regulations called "the Common Rule" governing human subject research sponsored by the federal government (NCPHS, 1979). The Common Rule was derived from the first four subparts of the DHHS regulations for the protection of human subjects. The Common Rule governs research that is conducted or supported by these federal agencies (45CFR46, 2001). The equivalent FDA human subject protection regulations govern research with drugs, biologics, and devices regardless of study sponsorship (21CFR50 \& 56, 2002). The Common Rule established three 
main protective mechanisms. They are (1) institutional assurances of compliance, (2) review of research by an institutional review board (IRB), and (3) requiring informed consent of subjects.

The integrity of scientific research in our society is monitored through the process of scientific review. Generally the scientific merit of research is determined by a system of peer review. However, not all research projects are subjected to peer review. In the early stages of a project, institutional funds, which may not be subject to an internal or external scientific review process, may be used to support the gathering of preliminary data. The review to ascertain if human subjects are being adequately protected in the conduct of a research study is done by an Institutional Research Board (IRB). The purpose of IRB is to review research and determine if the rights and welfare of human subjects involved in the research are adequately protected and to determine the risk-benefit ratio of the subject's participation in the study. Many institutions have policies that require research otherwise exempt from federal regulations follow the same rules for the protection of human subjects under the IRB system. The IRBs have the authority to approve, require modification in, or disapprove all research activities involving human subjects. Other authorities in the institution, even those that may administratively sit above the IRB leadership, cannot overrule the judgment of IRB to disprove or request modification of a research study. The IRBs must be allowed to function autonomously within an institution with respect to review of human subjects protocols in order to remain effective and unbiased.

Institutional Review Boards may be institutionally based, being primarily made up of faculty or staff of the institution, or they may be a private business fulfilling the review responsibility as stated by the federal regulations for a fee. In either situation the federal regulations dictate very specific rules for IRB membership and review procedures and documentation. The IRB member qualifications consider the experience and expertise of its members, their diversity (including race, gender, and cultural backgrounds), and their ability to represent the concerns of the scientific and lay community. These disparate voices are essential in ensuring that its advice properly safeguards the rights and welfare of human subjects. An IRB must consist of at least five members. Those members must include at least one scientist member, at least one nonscientist member, at least one noninstitutionally affiliated member, and one vulnerable subjects specialist, when research involving vulnerable subjects is under review. No IRB should consist entirely of men or entirely of women. Any member of IRB that is conflicted in any way with the study under consideration by IRB must recuse himself or herself from participation in the vote on that particular protocol (45CFR46, 2001).

There are essential elements that an IRB is required to consider for each study it reviews (Table II). The IRB must also monitor and investigate (1) any injuries to human subjects involved in protocols under their approval and (2) any serious or continuing noncompliance with regulations or requirements of IRB. The IRB is required to review each protocol under its authority at least once yearly, but more 
Table II. Essential Issues in an IRB Review

Determine risk to subjects and assess efforts within the research protocol to minimize these risks Analyze risk-benefit ratio, determine if it is reasonable

Ensure that the informed consent is comprehensive, properly obtained, and appropriately documented If appropriate, assess mechanisms for data safety monitoring

Ensure that selection of subjects is equitable

Assess how privacy and confidentiality of participants and their research findings will be protected Determine if research includes vulnerable subjects and how they will be protected

often if necessary to protect the safety of the human subjects involved. The process of IRB review may be institutionally specific, but because all reviews must follow the guidelines for structure and conduct outlined in the Code of Federal Regulations (45CFR46, 2001), the typical review process can be illustrated as outlined in Fig. 1.

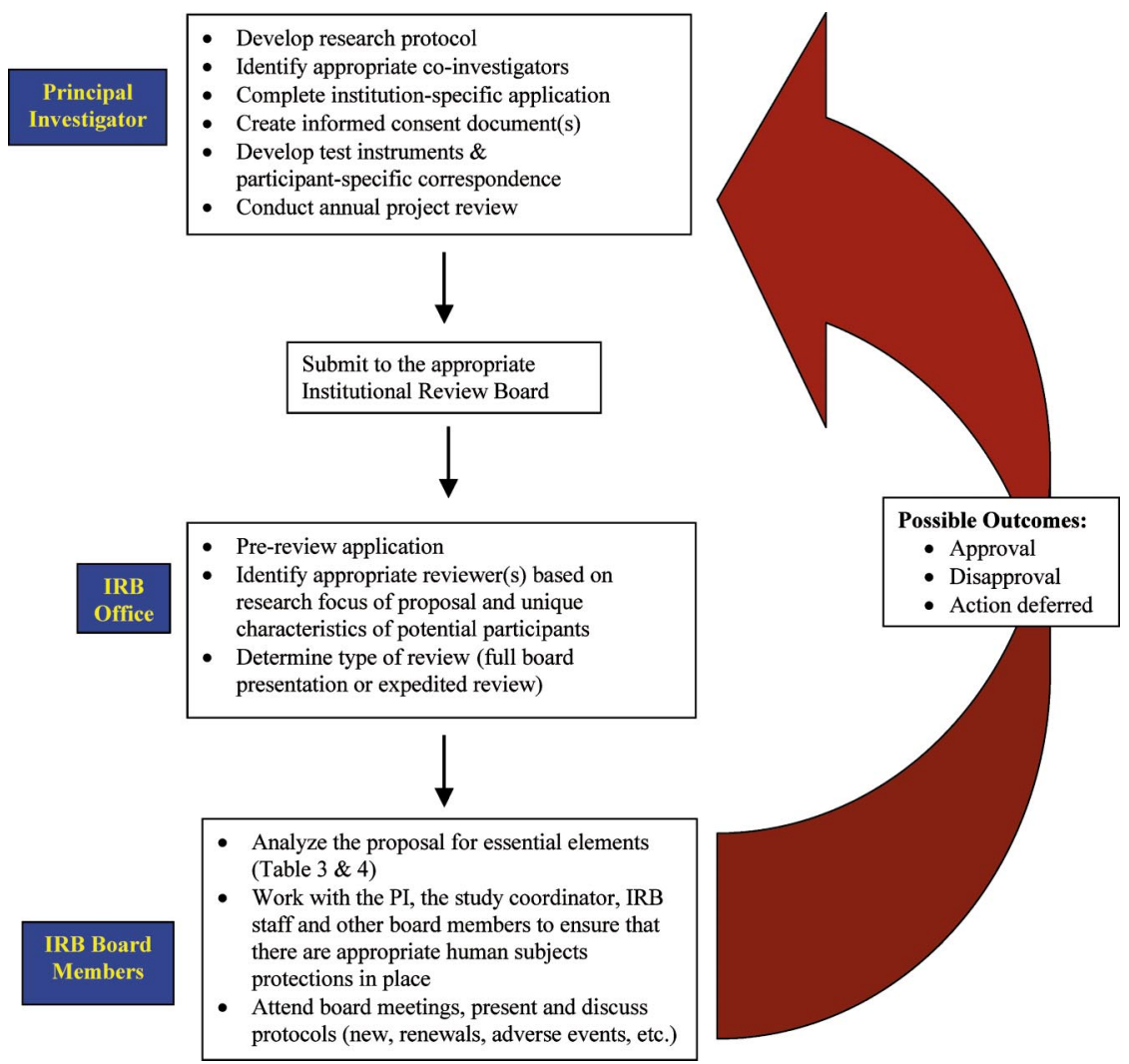

Fig. 1. Overview of the IRB research review process. 
Research can be exempt from ongoing IRB review if the research involves the collection or study of existing data, documents, records, pathological specimens, or diagnostic specimens, in which the data sources are either publicly available or the data was recorded by the investigator in such a manner that the subjects cannot be identified (either directly or indirectly through linked identifiers). Although additional categories of exempt research are identified in the federal regulations, these are less likely to occur in human genetic research studies (45CFR46, 2001). Only IRB has the power to declare a human subject research study exempt from ongoing review.

The Health Insurance Portability and Accountability Act (HIPAA) of 1996, also known as the "Privacy Rule," is a Federal regulation that protects certain health information and went into affect on April 14, 2002. Whereas the federal regulations regarding human subject research previous to HIPAA only protected human subjects who were by definition "living persons," the Privacy Rule was issued to protect the privacy of health information that identifies individuals who are either living or deceased. The HIPAA regulations apply to individually identifiable health information, known as "protected health information" (PHI) held or transmitted by a Covered Entity. Covered Entities as defined by the HIPAA rules include (1) health plans, (2) health care clearinghouses, and (3) health care providers who electronically transmit any health information in connection with transactions for which HHS has adopted standards. Generally these transactions concern billing and payment services or insurance coverage. Researchers are covered entities if they are also health care providers who electronically transmit health information. Researchers within a Covered Entity may be in a situation where the Privacy Rule applies to the entire entity including the research activity, or they may be excluded if their institution is classified as a Hybrid Entity where their research is defined as a noncovered function. Researchers should seek guidance from their own institutions as to the applicability of the HIPAA Privacy Rules to their institution and research.

The Privacy Rule does not replace or modify the Common Rule or FDA regulations, but adds additional privacy protections. Institutions may choose to have their IRB also serve the role of the HIPAA Privacy Board overseeing the application of the Privacy Rule to research at their institution or these can be established as separate review committees, each overseeing their respective applicable federal regulations. The information on the HIPAA Privacy Rule is lengthy and contains extensive detail about different research scenarios that are outside of what can be covered in this paper. Please see the Office of Civil Rights-HIPAA website for more information (Office of Civil Rights [OCR], 2003).

\section{Informed Consent}

The Belmont Report states that informed consent of human subjects in research must be (1) informed, (2) understood, and (3) voluntary. These are the principles of informed consent that provide respect for persons by honoring their 
autonomy and are intended to maximize the likelihood that consent is an informed autonomous decision. An appropriate informed consent document will provide subjects with all the information they need to make a decision, in a factual, complete, and accurate manner. The provision of informed consent is a process of information exchange between the investigator and the subject, and not simply obtaining a signature on the informed consent document. The burden is on the investigator to assure that the subject is truly informed about the study and freely choosing to participate. All subjects must have an opportunity to consider and consent/assent to participation not only on the initiation of a study, but also on an ongoing basis. Written documentation of consent is only required at the initiation of a study or when there are significant changes to the content of the consent document that may affect the subject's willingness to further participate.

When a child is the subject of research, that child is required to give "assent" to participation in addition to parental consent. Assent is defined as "a child's affirmative agreement to participate in research" (45CFR46, 2001) and a child's passive submission cannot be considered assent. For the child to be an active participant in the process, the "informed assent" must be tailored to consider the age, maturity, and mental ability of the child. The IRB is given wide latitude in determining whether a child is capable of assent and can waive this requirement if the child is incapable of providing assent, or if the research may offer direct benefits, or under the same conditions in which waiver of informed consent is granted to adult participants.

The Informed Consent Document is one of the most important tools in the protection of human subjects; thus, during the IRB review, this document is carefully evaluated. The Informed Consent Document and informed consent process must contain the basic essential elements described in Table III. To assure the document is understandable to the subject, it should be at a moderate reading level, avoid technical language when possible, and be in a language understandable to the subject. To ensure that the research participants are able to make an informed decision about their participation, the document should provide readers with a full understanding of the reasons, risks, and requirements of participation. Genetic counselors referring patients to researchers for involvement in studies should question an informed consent document that does not provide clear answers to all pertinent questions concerning the research under consideration.

The federal regulations allow particular situations where informed consent and/or the documentation of informed consent can be waived (45CFR46, 2001). Such waivers of informed consent can only be granted by IRB and only under specific conditions. To grant a waiver of informed consent, the IRB must find that all of the following criteria are met: (1) the study involves "no more than minimal risk" (minimal risk means that the probability and magnitude of harm or discomfort anticipated in the research are not greater in and of themselves than those ordinarily encountered in daily life or during the performance of routine physical or psychological examinations or tests); (2) the waiver will not adversely affect subject's 
Table III. Elements of Informed Consent

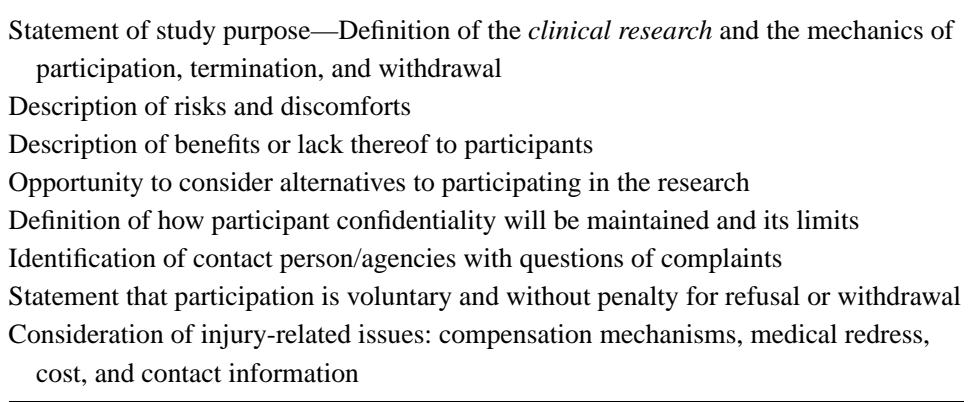

rights and welfare; (3) the research could not be practicably carried out without the waiver; and (4) when appropriate, the subjects will be given information after participation. The IRB approval for a study with waiver of informed consent, however, is still required. While all IRBs must find that a study meets all of the criteria listed above to be granted an informed consent waiver, often the issue raised in criterion \#3 regarding the practicability of doing the research without a waiver is up to IRB's subjective judgment. Whether the proposed subjects are available and reachable for informed consent to be obtained and how difficult it would be to reach them is discussed by IRB to determine practicability. Retrospective studies relying solely on the review of existing medical records, and where the privacy of the patient's identity and information is appropriately protected, may be granted a waiver of obtaining individual informed consent. However, if the IRB feels that the subject's rights could be harmed or that obtaining informed consent is practicable, they may decide that granting a waiver is not appropriate and require that full informed consent be obtained from each participating subject, before their existing data could be used in research. Granting of waivers of informed consent is determined by the individual IRBs through consideration of the federal regulations, the IRB's own policies, and the particulars of the proposed research.

\section{Informed Consent and HIPAA}

In addition to the requirement for informed consent, researchers under a Covered Entity using PHI are required to obtain a HIPAA Authorization from the patient to allow the use or disclosure of this information for research. Alternatively the researcher must be granted a waiver by their HIPAA Privacy Board or IRB to use the information without the patient's permission. The required elements of an authorization and the criteria for a waiver are stated in the HIPAA Privacy Rule (OCR, 2003). An institution may decide to have a document that combines the previously required elements of informed consent and the additional required HIPAA core elements and required statements for the HIPAA authorization incorporated 
into one document to be reviewed and signed by the research subject. The decision to have these as two separate or one combined document is decided at an institutional level. For more information about the required elements of a HIPAA Authorization, please see the Office of Civil Rights website (OCR, 2003).

\section{Human Genetic Research}

Although the guidelines for IRB review and attainment of informed consent (Tables II and III) are sufficiently broad to encompass human genetic research, there is concern that the potential risks to the subject in genetic studies may be underappreciated. Most human genetic research studies involve the use of DNA that is often obtained through a one-time blood or tissue collection and does not require ongoing subject participation. Because DNA studies are minimally invasive, the risks to the participant, based on the principles in the Belmont report, are minimal. However the traditional elements of an IRB review underestimate or ignore the nonphysical risks to the subject. The laws of inheritance and our society's views on the power of genetic information require that investigators and IRB reviewers consider additional issues in human subjects protection when considering human genetic research studies.

Genetic counselors are well aware of the nonphysical risks and benefits of genetic testing, including the impact on health and life insurance, employment, and psychosocial well-being. These same risks and benefits must also be considered when evaluating a human genetic research study to protect the involvement of human subjects. The ethical, legal, and social risks of participation in any research protocol should be fully explored and discussed with each subject during the consent process. The nonphysical risks associated with human genetic research should also be disclosed and considered in this discussion. Each of these additional areas will be briefly described below. Not all will pertain to every type of human genetic study, but each should be considered for applicability, and then, if applicable, the genetic counselor should be sure that the risks and benefits are covered in the research protocol and the informed consent document.

\section{Confidentiality and Security of Samples and Information}

The research protocol and the informed consent document should discuss the manner in which the DNA samples and information relating to the sample and participant will be stored during the study and at its completion. All research tissue/DNA or other identifiable research information should be kept secure and in a fashion that protects the identity of the subject. To protect the confidentiality and privacy of the subjects, DNA should be stored in a coded fashion, without attached identifiable information. If DNA or other information from the study will be shared with collaborators inside or outside the institution, the informed 
consent should describe this process and detail the mechanisms by which the confidentiality and privacy of the subject's information will be protected when information or DNA is shared. There should be provisions for how DNA or other information will be stored or disposed of after the study is over or if the researcher leaves their current institution. The informed consent should state if the tissue and information would be anonymized, destroyed, or maintained at that time. The use of samples or information that are anonymous are encouraged wherever such research is possible. Anonymous means that there is no code or link on physical samples or information associated with them that can be connected back to the identity of the subject. Anonymous research affords the most protection to human subjects, but is not always possible or practical. In addition, this type of research has the least benefit because the study design makes it impossible to share individual results with subjects. If the tissue or information is to be maintained, the protections and limitations of the storage and potential future use need to be explained and the subject should be given the option of choosing whether or not to participate further (University of Michigan Medical School Institutional Review Board, 2002). The issue of future secondary use will be more fully addressed below.

\section{Family Issues in Genetic Research}

Because of the heritable nature of many genetic diseases and conditions, many genetic research studies require the involvement of related individuals and/or entire families. If the research under consideration does involve information about family members, particular attention needs to be taken to protect all individuals involved (American Society of Human Genetics [ASHG], 2000). This is of particular concern when information is gathered about a family member without their knowledge and without their direct consent. Such information can typically be found in a family medical history or pedigree. When an individual's sensitive or private information is included in research without obtaining their direct consent, their privacy should be protected by coding the information such that identifiable information is not easily linked to the identity of the individual. The research should take care to only collect the information needed to conduct the study and not extra identifiable information that could pose harm or embarrassment to the nonconsented family members. If identifying medical or private information is collected through a second party, the investigator should try to get direct informed consent from the individual to whom the information belongs (Botkin, 2001). In situations where this is not practicable the investigator should request an IRB waiver of informed consent for the inclusion of information from individuals that have not directly consented to the use of their private information in the research study (ASHG, 2000).

The informed consent should state what precautions are in place to protect the privacy and confidentiality of subjects and families when information is published 
or publicly presented. Be mindful of the fact that for rare disorders or families with unique structure, publication or presentation of the pedigree or situation may directly identify the family or individual even without the direct disclosure of other identifiable information.

The investigator should have a plan stating how family members will be contacted if they are to be recruited for participation in the research study. Investigators should not directly contact family members unknown to them, because doing so could be seen as a breach of privacy. Family members already in contact with the investigator can provide information about the study to their relatives, who would then have the choice to contact the investigators if they are interested in participating. Care should be taken to ensure that relatives are not forced or coerced into participating in a research study. Likewise, physicians or genetic counselors referring patients or families of interest to the research team should serve as a contact to the family or subjects and not give the research team contact information for subjects that have not consented to be contacted for research participation.

\section{Results of the Research}

Many genetic research studies have the potential to provide a family or individuals research results that could provide a genetic diagnosis or impact risk predictions. If such results are possible, the research team should decide if the results have the credibility and value to warrant disclosure to the subject. A riskbenefit analysis should be undertaken to analyze this issue. If the research results are deemed appropriate to be shared with the subject, the subject should be given the choice to accept or decline this opportunity after receiving full disclosure of the uncertainties or weaknesses of the research information, as well as the potential risks and benefits. It is strongly encouraged that the disclosure of genetic research results is accompanied by appropriate genetic counseling, which will help subjects gain a full understanding of the significance and limitations of the research findings. If genetic counseling is required to obtain research results, the subject must be informed of the potential cost of counseling during the informed consent process.

Additional protections should be considered to protect the confidentiality of the research information, such as exemption from placing research results in the clinical medical record. If the research information will be used to make future medical decisions, extreme care should be taken to assure the value and reliability of the research result. Subjects receiving research results should be encouraged to have these results reconfirmed when/if clinically certified tests for the disease or condition become available. In studies where researchers have decided not to share research results with the subject, a plan should be developed that explains when and if the research team would be compelled to disclose research information. Such a situation could arise if the research team should unexpectedly encounter 
information about the subject in the conduct of the research that is of such medical importance that withholding the information from the subject would be harmful or unethical.

\section{Secondary Use of Samples or Information}

The informed consent should specifically describe if there are other research studies in which the subject's information and/or tissue (DNA) may be used. If the researcher plans to utilize the information or tissue for future research studies, this needs to be disclosed and consented to by the subject. If the tissue and information are to be used in future research in an identifiable manner, consent from the subject should be obtained that is as specific as possible to the future intended uses. Identifiable means that linkage between the sample and/or information and the identity of the subject is possible. The subject should be allowed to decide if they are willing to have their tissue or information used for future studies and what the limitations of such future use will be, such as anonymous use only or use for only certain related research studies. The subject should also be able to state if he/she requires recontact and reconsent for the future utilization of their tissue or information in research. Even with prior consent for future use of the tissue or information by the subject, the researcher is still required to get separate approval for any future or additional studies by IRB. In the review of the new study, the IRB will decide if the prior consent of the subject obtained under a previous research study is sufficient for the use of the tissue or DNA in the new study under consideration.

\section{Additional Issues in Human Genetic Research}

The protocol and informed consent document should discuss social, psychological, or emotional issues that may result from the research, such as issues of parentage, predictive disease or risk information, and impact on insurability, employability, or other social harms. These issues should be considered both if unintentional discovery of the research information by the subject or others should occur, and if direct disclosure of the research results is an option of the study (see above). Careful consideration should also be undertaken to protect the privacy of human subjects in genetic research studies where simply being involved in the research alone could label the subject and be harmful. In this situation, the research team should request that information that identifies the subject as a research participant is carefully protected and is not included or mentioned in the subject's clinical medical record. Researchers should take care when allowing the inclusion of informed consent documents in the subject's medical record because of this issue of identification. For some studies in which particularly sensitive information is collected or generated in the course of the research, the research team may want to apply for a Certificate of Confidentiality (CoC). A CoC allows the 
researchers to further protect the privacy of research subjects by allowing them to withhold subject's names or other identifying information from courts or governmental agencies, even if subpoenaed to disclose this information. The OHRP website contains guidance about the use of CoCs and links to a list of contacts at different federal agencies for information about obtaining these certificates (OHRP, 2003a)

Withdrawal from genetic research poses its own unique problems. Because the involvement of a subject in a DNA-based genetic study does not require their ongoing physical involvement, withdrawal may be complicated. If DNA and information has not yet been utilized in the study, then the subject could request his tissue and data be destroyed and not utilized in the research. If DNA and information have already been used in the research analysis, full withdrawal may be impossible and only DNA remaining in storage may be able to be withdrawn and destroyed, but not DNA and data already utilized. When an entire family or related individuals are participants in research, withdrawal from the study is further complicated by the genetic relatedness of the subjects in the family. Even if an individual withdraws or chooses not to participate in the research, information may still be discovered about them because of their relationship to others in the study. Researchers and IRBs should always be cognizant of these possibilities and in the informed consent document and process the subject needs to be notified if there are limitations on the ability to withdraw from the research.

\section{The Role of the Genetic Counselor}

The practice of genetic counseling is intertwined with the conduct of human genetic research. The field of human genetics is on the cutting edge of biomedical technology, with discoveries in the laboratory finding their way into the clinic in the form of research studies often before genetic testing or therapies are commercially available. Genetic counselors need to assist their patients in understanding the complex issues in genetic research and in making choices that allow for full autonomy and informed consent. Genetic counselors must help patients to understand that the primary goal of research is to gain generalizable information and not to provide individual benefits to participants. Benefits that may arise from participation in research need to be evaluated in terms of quality, applicability, and utility as well as the potential for harm. For a genetic counselor to fulfill this aspect of their role, they must be educated about the rules and regulations that govern the conduct of human subject research and must assist their patients (subjects) in understanding the study in its entirety, including the informed consent document pertaining to the research under consideration. The genetic counselor is uniquely positioned to play an important role in understanding the nuances of the genetic research interface between basic/clinical science and genetic counseling. 
The genetic counselor must keep their role as an advocate for their patients clear when being asked to facilitate the progress of genetic research. Although the research goals may be valid and important, the clinical genetic counselor must ask for and obtain complete information about the research project to help inform their patients about participation in the research and the benefits and risks that may be involved. Genetic counselors playing dual roles, as members of research teams and also as genetic counselors to subjects in the research studies, must take particular care to be sure they are clear as to what "hat" they are wearing at any one time. Such dual situations should be avoided whenever possible so there are no real or perceived conflicts of interest on the part of the genetic counselor involved (Association of American Medical Colleges, 2002).

The best practice of genetic counseling when interfacing with the conduct of human subject research will be provided by a genetic counselor who is knowledgeable about the ethical principles and regulations that govern human subject research and who is also aware of the special issues or considerations that are important to human genetic research. Genetic counselors are particularly skilled at providing clear information to patients and families about complicated genetic principles and issues. This ability also makes genetic counselors uniquely qualified to assist in the informed consent and education of subjects considering participation in human genetic research studies.

\section{REFERENCES}

American Society of Human Genetics. (2000). Genetic policy statements. Family history and privacy advisory. Retrieved from www.faseb.org/genetics/ashg/policy/pol-38.htm

Association of American Medical Colleges. (2002). Protecting subjects, preserving trust, promoting progress II: Principles and recommendations for oversights of an institution's financial interests in human subjects research. Retrieved from www.aamc.org/members/coitf/2002coireport.pdf

Botkin, J. R. (2001). Protecting the privacy of family members in survey and pedigree research. JAMA, 285, 207-211.

Code of Federal Regulations. Title 21, Parts 50 and 56 (21 CFR 50 \& 56). (2002). FDA Regulations. Retrieved http://www.access.gpo.gov/nara/cfr/waisidx_03/21cfr5_03.html

Code of Federal Regulations. Title 21, Part 312 (21 CFR 312). (2002). Investigational new drug application. Retrieved from http://www.access.gpo.gov/nara/cfr/waisidx_03/21cfr312_03.html

Code of Federal Regulations. Title 45, Part 46 (45 CFR 46). (2001). DHHS protection of human subjects. Retrieved from http://www.access.gpo.gov/nara/cfr/waisidx_02/45cfr46_02.html

International Conference on Harmonization of Technical Requirements for Registration of Pharmaceuticals for Human Use (ICH). (1996). ICH Harmonised Tripartite Guideline: Guideline for good clinical practice (E6). Retrieved from http://www.ich.org/ MediaServer.jser? @_ID=482\&@_MODE=GLB

National Commission for the Protection of Human Subjects of Biomedical and Behavioral Research. (1979). The Belmont Report: Ethical principles and guidelines for the protection of human subjects of research. Retrieved from http://ohrp.osophs.dhhs.gov/humansubjects/guidance/belmont.htm

National Institutes of Health. (2001).Required education in the protection of human research participants (NOT-OD-01-061). Retrieved from http://grants1.nih.gov/grants/guide/notice-files/NOTOD-01-061.html

National Society of Genetic Counselors. (1992). Code of ethics. Retrieved from http://www.nsgc.org

National Society of Genetic Counselors. (2002). Professional Status Survey 2002. Retrieved from http://www.nsgc.org/pdf/PSS_2002_2_22.pdf 
Office of Civil Rights. (2003). Department of Health and Human Services, Office of Civil Rights HIPAA Homepage. Retrieved from http://www.hhs.gov/ocr/hipaa/

Office of Human Research Protections. (2003a). Guidance on certificates of confidentiality. Retrieved from http://ohrp.osophs.dhhs.gov/humansubjects/guidance/certconf.htm

Office of Human Research Protections. (2003b). OHRP compliance activities determination letters. Retrieved from http://ohrp.osophs.dhhs.gov/detrm_letrs/lindex.htm

University of Michigan Medical School Institutional Review Board. (2002). IRBMED Guidance for $I R B$ reviewers and medical school investigators regarding genetic/DNA research studies. Retrieved from http://www.med.umich.edu/irbmed/InformationalDocuments/GeneticAnalysis.pdf

World Medical Association. (2002). Declaration of Helsinki. Ethical principles for medical research involving human subjects. Retrieved from http://www.wma.net/e/policy/b3.htm 